\title{
COMPARISON OF THE SINGULAR NUMBERS OF CORRECT RESTRICTIONS OF ELLIPTIC DIFFERENTIAL OPERATORS
}

\author{
V. I. BURENKOV AND M. OTELBAEV
}

\begin{abstract}
The paper is dedicated to finding the asymptotics of singular numbers of a correct restriction of a uniformly elliptic differential operator of order $2 l$ defined on a bounded domain in $\mathbb{R}^{n}$ with sufficiently smooth boundary, which is in general a nonselfadjoint operator. Conditions are established on a correct restriction, ensuring that its singular numbers $s_{k}$ are of order $k^{2 l / n}$ as $k \rightarrow \infty$. As an application of this result certain estimates are obtained for the deviation upon domain perturbation of singular numbers of such correct restrictions.
\end{abstract}

\section{$\S 1$. INTRODUCTION}

Let $l, n \in \mathbb{N}$ and $\mathcal{L}$ be an elliptic differential expression of the following form: for $u \in C^{\infty}\left(\mathbb{R}^{n}\right)$

$$
(\mathcal{L} u)(x)=\sum_{|\alpha|,|\beta| \leq l}(-1)^{|\alpha|+|\beta|} D^{\alpha}\left(A_{\alpha \beta}(x) D^{\beta} u\right), \quad x \in \mathbb{R}^{n},
$$

where $A_{\alpha \beta} \in C^{l}\left(\mathbb{R}^{n}\right)$ are real-valued functions for all multi-indices $\alpha, \beta$ satisfying $|\alpha|,|\beta|$ $\leq l$. Moreover, for any bounded domain $\Omega \subseteq \mathbb{R}^{n}$ with sufficiently smooth boundary let $L_{\Omega}$ be a linear operator closed in $L_{2}(\Omega)$ generated by $\mathcal{L}$ on $\Omega$.

Our main aim is finding a family of correct restrictions $B$ of the operator $L_{\Omega}$ having the same asymptotics of singular numbers $s_{k}(B)$.

This is done by comparing $s_{k}(B)$ with singular numbers $s_{k}(A)$ of a correct restriction $A$ of the operator $L_{\Omega}$ whose asymptotics of singular numbers are known and represent the inverse $B^{-1}$ in the form $B^{-1}=A^{-1}+K$, where $A^{-1}$ and $K$ are a leading operator and a non-leading operator respectively.

As an application we establish certain estimates for the deviation of singular numbers $s_{k}(B)$ of correct restrictions of this family upon domain perturbation. This is done by considering a correct restriction $A$ for which such estimates are known and the fact that $s_{k}(B)$ and $s_{k}(A)$ have the same asymptotics which are uniform with respect to a certain family of correct restrictions and a certain class of domains $\Omega$.

The paper is organized in the following way. In Section 2 we recall the notion of a correct restriction of a closed linear operator in a Hilbert space and related facts. In Section 3 the definition of leading and non-leading compact operators is given and, under certain assumptions, it is proved that the singular numbers of the sum of a leading and a non-leading operator have the same asymptotics as the singular numbers of the leading operator (Theorem 3.1). Section 4 is dedicated to some auxiliary estimates of the singular numbers of correct restrictions of elliptic differential operators. In Section 5

2010 Mathematics Subject Classification. Primary 35P15, 35P20, 35J40, 47A75.

Key words and phrases. Correct restrictions of operators, leading and non-leading operators, estimates and asymptotics for singular numbers, spectral stability estimates.

V. I. Burenkov's research was supported by a grant from the Russian Scientific Foundation (project 14-11-00443). 
a precise description of the class of domains under consideration is given. The core result on the representation of the inverse of a correct restriction as the sum of a leading and a non-leading operator is proved in Section 6 (Theorem 6.1). In Section 7 on the basis of Theorems 3.1 and 6.1 it is proved that the singular numbers of all correct restrictions of a certain family are of the same order $k^{2 l / n}$ and have the same asymptotics as $k \rightarrow \infty$ (Theorem 7.1). Section 8 is dedicated to applying Corollary 7.1 (a "uniform" version of Theorem 7.1) to obtain spectral stability estimates for correct restrictions belonging to a certain family (Theorem 8.1).

Most of the results of this paper were formulated, without proofs, in [4].

\section{$\S$ 2. Preliminaries on CORRect Restrictions of Linear operators}

Let $L: D(L) \rightarrow H$ be a closed linear operator in a Hilbert space $H$. It will always be assumed that the domain $D(L)$ is dense in $H$ and that the equation

$$
L u=f
$$

has a solution for any $f \in H$ (in general non-unique).

Recall that an operator $A: D(A) \rightarrow H$ is called a restriction of $L$, briefly $A \subseteq L$, if $D(A) \subseteq D(L)$ and $A u=L u$ for all $u \in D(A)$.

Definition 2.1. A restriction $A$ of $L$ is called correct if the equation

$$
A u=f
$$

has a unique solution $u \in D(A)$ for any $f \in H$ and the corresponding inverse operator $A^{-1}: H \rightarrow D(A)$ is bounded, i. e.

$$
\left\|A^{-1}\right\|=\sup _{f \in H, f \neq 0} \frac{\left\|A^{-1} f\right\|}{\|f\|}=\sup _{u \in D(A), u \neq 0} \frac{\|u\|}{\|A u\|}<\infty .
$$

Remark 2.1. Clearly $L A^{-1}=I$, where $I$ is the identity operator on $H$. Moreover, if $B: H \rightarrow D(L)$ is a bounded operator such that $L B=I$, then the operator $A$ which is the restriction of $L$ to $D(A)=B(H)$ is a correct restriction of $L$ and $B=A^{-1}$.

Let $L$ have a correct restriction $A$. Then it is possible to describe all correct restrictions of $L$. Namely, the following statement holds. See [9, 11].

Lemma 2.1. Let $A$ be a correct restriction of $L$.

a) If an operator $K: H \rightarrow \operatorname{Ker} L=\{u \in D(L): L u=0\}$ is bounded, then the operator $A^{-1}+K$ is invertible and the operator

$$
B=\left(A^{-1}+K\right)^{-1}
$$

is a correct restriction of $L$.

b) If $B$ is a correct restriction of $L$, then there exists a bounded operator

$$
K: H \rightarrow \operatorname{Ker} L,
$$

namely $K=B^{-1}-A^{-1}$, such that equality (3) holds.

Remark 2.2. Under the assumptions of part a)

$$
B^{-1}=A^{-1}+K
$$

and

$$
D(B)=R\left(A^{-1}+K\right) \subseteq D(A)+R(K) \subseteq D(A)+\text { Ker } L .
$$

Remark 2.3. In Lemma 2.1 the operators $K$ can be nonlinear, which allows us to consider boundary value problems with nonlinear boundary conditions. However, in this paper we restrict ourselves to considering the case of linear operators $K$. 
Example 2.1. Let $B$ be an open ball in $\mathbb{R}^{n}$ and $L$ be the closure of the Laplacian $-\Delta$ with the domain $C^{\infty}\left(\mathbb{R}^{n}\right)$ in $H=L_{2}(B)$; hence $L: D(L) \rightarrow L_{2}(B)$,

$$
D(L)=\left\{u \in L_{2}(B):-\Delta_{w} u \in L_{2}(B)\right\}, \quad L u=-\Delta_{w} u \quad \text { for } u \in D(L),
$$

where $-\Delta_{w} u$ is the weak Laplacian.

Furthermore, in the capacity of a restriction $A$ of $L$, we consider the Dirichlet Laplacian $-\Delta_{\mathrm{D}}$ with the domain

$$
D\left(-\Delta_{\mathrm{D}}\right)=\left\{u \in D(L), \operatorname{tr}_{\partial B} u=0\right\} .
$$

It is well known that for each $f \in L_{2}(B)$ the equation $-\Delta_{\mathrm{D}} u=f$ has a unique solution in $D\left(-\Delta_{\mathrm{D}}\right)$ which continuously depends on $f$. Hence $-\Delta_{\mathrm{D}}$ is a correct restriction of $L$ in the sense of Definition 1.

By Lemma 2.1 for each bounded operator $K: L_{2}(B) \rightarrow \operatorname{Ker} L$, the space of all solutions $u \in D(L)$ of the equation $\Delta_{w} u=0$, and the operator $\left(\left(-\Delta_{\mathrm{D}}\right)^{-1}+K\right)^{-1}$ is a correct restriction of $L$. This implies that for each $f \in L_{2}(B)$ the equation

$$
\left(\left(-\Delta_{\mathrm{D}}\right)^{-1}+K\right)^{-1} u=f
$$

has a unique solution

$$
\begin{aligned}
& u=\left(\left(-\Delta_{\mathrm{D}}\right)^{-1}+K\right) f \in D\left(\left(\left(-\Delta_{\mathrm{D}}\right)^{-1}+K\right)^{-1}\right)=R\left(\left(-\Delta_{\mathrm{D}}\right)^{-1}\right)+R(K) \\
& \subseteq D\left(-\Delta_{\mathrm{D}}\right)+R(K) \subseteq D\left(-\Delta_{\mathrm{D}}\right)+\operatorname{Ker} L .
\end{aligned}
$$

Moreover,

$$
\left\{\begin{array} { l } 
{ u \in D ( ( ( - \Delta _ { \mathrm { D } } ) ^ { - 1 } + K ) ^ { - 1 } ) , } \\
{ ( ( - \Delta _ { \mathrm { D } } ) ^ { - 1 } + K ) ^ { - 1 } u = f }
\end{array} \Leftrightarrow \left\{\begin{array}{l}
u \in L_{2}(B),-\Delta_{w} u \in L_{2}(B), \\
-\Delta_{w} u=f \text { on } B, \\
\operatorname{tr}_{\partial B}\left(u+K\left(\Delta_{w} u\right)\right)=0 .
\end{array}\right.\right.
$$

The implication $\Rightarrow$ follows since by (6) and (7) $-\Delta_{w} u=f$ on $\Omega$ and

$$
\operatorname{tr}_{\partial B} u=\operatorname{tr}_{\partial B}\left(-\Delta_{\mathrm{D}}\right)^{-1} f+\operatorname{tr}_{\partial B} K f=\operatorname{tr}_{\partial B} K f .
$$

Conversely, assume that $u \in L_{2}(B),-\Delta_{w} u \in L_{2}(B),-\Delta_{w} u=f$ on $B, \operatorname{tr}_{\partial B} u=\operatorname{tr}_{\partial B} K f$, and $v=u-K f$. Then $v \in D(L)$ (since $K f \in D(L)),-\Delta_{w} v=f$ on $B$, and $\operatorname{tr}_{\partial B} v=0$. Therefore, $v=\left(-\Delta_{\mathrm{D}}\right)^{-1} f$. Consequently,

$$
u=\left(-\Delta_{\mathrm{D}}\right)^{-1} f+K f \Leftrightarrow u \in R\left(\left(-\Delta_{\mathrm{D}}\right)^{-1}+K\right)=D\left(\left(\left(-\Delta_{\mathrm{D}}\right)^{-1}+K\right)^{-1}\right)
$$

and $\left(\left(-\Delta_{\mathrm{D}}\right)^{-1}+K\right)^{-1} u=f$.

Example 2.2. Let $H, L, A$ be the same as in Example 2.1, $n \geq 2$ and $\varphi_{1}, \varphi_{2} \in L_{2}(B)$. Assume that $\varphi_{1}$ is a harmonic function on $B$ and that for all $f \in L_{2}(B)$

$$
(K f)(x)=\varphi_{1}(x) \int_{B} \varphi_{2}(y) f(y) d y, \quad x \in B .
$$

Then $K$ is bounded from $L_{2}(B)$ to $\operatorname{Ker} L$, and all the statements of Example 2.1 are applicable to $K$. In particular this implies that the following problem

$$
\left\{\begin{array}{l}
u \in L_{2}(B),-\Delta_{w} u \in L_{2}(B), \\
-\Delta_{w} u=f \text { on } B \\
\operatorname{tr}_{\partial B}\left(u(x)+\varphi_{1}(x) \int_{B} \varphi_{2}(y) \Delta_{w} u(y) d y\right)=0
\end{array}\right.
$$

with a non-local boundary condition is well posed. 


\section{$\S$ 3. Some estimates FOR SINGUlar numbers OF GENERAL COMPACT LINEAR OPERATORS}

Let $A$ be a compact linear operator in a Hilbert space $H$. Recall that the eigenvalues of the self-adjoint operator $\left(A^{*} A\right)^{1 / 2}$ are called the singular numbers of the operator $A$. As usual all singular numbers of $A$ will be written in the form of the sequence $\left\{s_{n}(A)\right\}_{n=1}^{\infty}$, where

$$
s_{1}(A) \geq s_{2}(A) \geq \cdots \geq s_{n}(A) \geq \cdots
$$

and each singular number is repeated as many times as its multiplicity. Note that $\lim _{n \rightarrow \infty} s_{n}(A)=0$.

Lemma 3.1. Let $A$ and $B$ be compact linear operators in a Hilbert space $H, 0<\alpha<\beta$ and $\alpha / \beta<\theta<1$.

Moreover, let for some $c_{1}, c_{2}>0$ for all $k \in \mathbb{N}$

$$
c_{1} k^{-\alpha} \leq s_{k}(A), \quad s_{k}(B) \leq c_{2} k^{-\beta} .
$$

Then for all $k \in \mathbb{N}$

$$
s_{k}(A+B) \leq s_{k-\left[k^{\theta}\right]+1}(A)\left(1+c_{3} k^{\alpha-\theta \beta}\right),
$$

where $c_{3}=c_{1}^{-1} c_{2} 2^{\beta}$ and $[a]$ denotes the integer part of a real number $a$.

Proof. We apply the inequality

$$
s_{k}(A+B) \leq \min _{\substack{m+n=k+1 \\ m, n \in \mathbb{N}}}\left(s_{m}(A)+s_{n}(B)\right) .
$$

([5, 6, 7]; see also [8, Chapter 2, Corollary 2.2].)

Taking into account that $\frac{\mu}{[\mu]} \leq 2$ for each real number $\mu \geq 1$, we have

$$
\begin{aligned}
& s_{k}(A+B) \leq s_{k-\left[k^{\theta}\right]+1}(A)+s_{\left[k^{\theta}\right]}(B) \\
& \quad \leq s_{k-\left[k^{\theta}\right]}(A)+c_{2}\left[k^{\theta}\right]^{-\beta} \frac{s_{k-\left[k^{\theta}\right]+1}(A)}{c_{1}\left(k-\left[k^{\theta}\right]+1\right)^{-\alpha}} \leq s_{k-\left[k^{\theta}\right]}(A)\left(1+c_{1}^{-1} c_{2}\left[k^{\theta}\right]^{-\beta} k^{\alpha}\right) \\
& \quad \leq s_{k-\left[k^{\theta}\right]+1}(A)\left(1+c_{1}^{-1} c_{2}\left(\frac{k^{\theta}}{\left[k^{\theta}\right]}\right)^{\beta} k^{\alpha-\theta \beta}\right) \leq s_{k-\left[k^{\theta}\right]}(A)\left(1+c_{1}^{-1} c_{2} 2^{\beta} k^{\alpha-\theta \beta}\right) .
\end{aligned}
$$

Hence, inequality (10) follows.

Remark 3.1. Assume that for some $k_{1} \in \mathbb{N}$ inequalities (9) are satisfied with the same $c_{1}, c_{2}$ only for natural $k \geq k_{1}$. Since $\left[k^{\theta}\right], k-\left[k^{\theta}\right] \rightarrow \infty$ as $k \rightarrow \infty$, there exists $k_{2} \in \mathbb{N}$, depending only on $k_{1}$ and $\theta$, such that $\left[k^{\theta}\right], k-\left[k^{\theta}\right]+1 \geq k_{1}$ for all $k \geq k_{2}$. Hence, by the above proof, it follows that inequality (10) is satisfied with the same $c_{3}$ for natural natural $k \geq k_{2}$.

Lemma 3.2. Under the assumptions of Lemma 3.1 there exists $c_{4}>0$, depending only on $\alpha, \beta, \theta, c_{1}, c_{2}$ and for each $\gamma>1$ there exists $\varkappa_{\gamma} \in \mathbb{N}$, depending only on $\gamma, \alpha, \beta, \theta, c_{1}, c_{2}$ such that

$$
s_{k+\left[\gamma k^{\theta}\right]}(A)\left(1-c_{4} k^{\alpha-\theta \beta}\right) \leq s_{k}(A+B) \leq s_{k-\left[k^{\theta}\right]}(A)\left(1+c_{4} k^{\alpha-\theta \beta}\right)
$$

for all $k \geq \varkappa_{\gamma}$.

Proof. 1. By inequality (11), with $B$ replaced by $-B, A$ replaced by $A+B$, and $k$ replaced by $2 k$, we have

$$
s_{2 k}(A) \leq s_{k}(A+B)+s_{k+1}(B) .
$$

Hence, by assumption (9)

$s_{k}(A+B) \geq s_{2 k}(A)-s_{k+1}(B) \geq c_{1}(2 k)^{-\alpha}-c_{2}(k+1)^{-\beta} \geq k^{-\alpha}\left(c_{1} 2^{-\alpha}-c_{2} k^{\alpha-\beta}\right) \geq c_{5} k^{-\alpha}$, 
where $c_{5}=c_{1} 2^{-\alpha-1}$, if $k \geq k_{1}=\left[\left(c_{1}^{-1} c_{2} 2^{\alpha+1}\right)^{1 /(\beta-\alpha)}\right]+1$.

Therefore by Lemma 3.1 and Remark 3.1 it follows that there exists $k_{2} \in \mathbb{N}$, depending only on $\alpha, \beta, \theta, c_{1}, c_{2}$, such that inequality (10) holds, with $B$ replaced by $-B, A$ replaced by $A+B, k$ replaced by $m$, and $c_{1}$ replaced by $c_{5}$, for all $m \geq k_{2}$. So

$$
s_{m}(A) \leq s_{m-\left[m^{\theta}\right]+1}(A+B)\left(1+c_{4} m^{\alpha-\theta \beta}\right),
$$

where $c_{4}=c_{5}^{-1} c_{2} 2^{\beta}>c_{3}$, for all $m \geq k_{2}$.

2. For a given $k \in \mathbb{N}$, let a natural number $m$ be such that

$$
m-\left[m^{\theta}\right]+1=k .
$$

2a. Note that the function $f$ defined by $f(m)=m-\left[m^{\theta}\right]+1$ for $m \in \mathbb{N}$ is nondecreasing on $\mathbb{N}$ and $f(\mathbb{N})=\mathbb{N}$. Therefore this equation has a solution for each $k \in \mathbb{N}$, although the solution, in general, is non unique.

Indeed, first note that for all $m, n \in \mathbb{N}$ the inequalities

$$
n-1<m^{\theta} \leq n, \quad(n-1)^{1 / \theta}<m^{\theta} \leq n^{1 / \theta} \quad \text { and } \quad\left[(n-1)^{1 / \theta}\right]+1 \leq m \leq\left[n^{1 / \theta}\right]
$$

are equivalent. Hence

$$
\begin{aligned}
f(\mathbb{N}) & =\bigcup_{n=1}^{\infty} f\left(\left\{m \in \mathbb{N}: n-1<m^{\theta} \leq n\right\}\right) \\
& =\bigcup_{n=1}^{\infty} f\left(\left\{m \in \mathbb{N}:\left[(n-1)^{1 / \theta}\right]+1 \leq m \leq\left[n^{1 / \theta}\right]\right\}\right) .
\end{aligned}
$$

Assume that $n$ is fixed and $\left[(n-1)^{1 / \theta}\right]+1<\left[n^{1 / \theta}\right]$. If $\left[(n-1)^{1 / \theta}\right]+1 \leq m^{\theta} \leq\left[n^{1 / \theta}\right]-1$, then $n-1<m^{\theta}<n$; hence $\left[m^{\theta}\right]=n-1$ and

$$
f(m)=m-\left[m^{\theta}\right]+1=m-n+2 .
$$

If $n^{1 / \theta} \notin \mathbb{N}$, then $n-1 \leq\left(n^{1 / \theta}-1\right)^{\theta}<\left[n^{1 / \theta}\right]^{\theta}<n$ hence $\left[\left[n^{1 / \theta}\right]^{\theta}\right]=n-1$, and this equality also holds for $m=\left[n^{1 / \theta}\right]$. If $n^{1 / \theta} \notin \mathbb{N}$, then $\left[n^{1 / \theta}\right]^{\theta}=n$ and

$$
f\left(\left[n^{1 / \theta}\right]\right)=\left[n^{1 / \theta}\right]-n+1=f\left(\left[n^{1 / \theta}\right]-1\right) .
$$

In both cases

$$
f\left(\left\{m \in \mathbb{N}:\left[(n-1)^{1 / \theta}\right]+1 \leq m \leq\left[n^{1 / \theta}\right]\right\}\right)=\left[f\left(\left[(n-1)^{1 / \theta}\right]+1\right), f\left(\left[n^{1 / \theta}\right]\right)\right] \cap \mathbb{N} .
$$

Clearly this equality is also satisfied if $\left[(n-1)^{1 / \theta}\right]+1=\left[n^{1 / \theta}\right]$.

Therefore

$$
\begin{aligned}
f(\mathbb{N}) & =\bigcup_{n=1}^{\infty}\left(\left[f\left(\left[(n-1)^{1 / \theta}\right]+1\right), f\left(\left[n^{1 / \theta}\right]\right)\right] \cap \mathbb{N}\right) \\
& =\left(\bigcup_{n=1}^{\infty}\left[f\left(\left[(n-1)^{1 / \theta}\right]+1\right), f\left(\left[n^{1 / \theta}\right]\right)\right]\right) \cap \mathbb{N}=\mathbb{N}
\end{aligned}
$$

because $f(1)=1$ and for each $n \in \mathbb{N}$

$$
f\left(\left[n^{1 / \theta}\right]+1\right)=\left[n^{1 / \theta}\right]-\left[\left(\left[n^{1 / \theta}\right]+1\right)^{\theta}\right]+2 \leq\left[n^{1 / \theta}\right]-\left[\left(\left[n^{1 / \theta}\right]\right)^{\theta}\right]+2=f\left(\left[n^{1 / \theta}\right]\right)+1 .
$$

Also note that

$$
f\left(\left[n^{1 / \theta}\right]+1\right) \geq\left[n^{1 / \theta}\right]-\left[\left(\left[n^{1 / \theta}\right]\right)^{\theta}+1\right]+2=f\left(\left[n^{1 / \theta}\right]\right) .
$$

The above argument also implies that the function $f$ is non-decreasing on $\mathbb{N}$, but is not strictly increasing.

2b. Assume that $m \in \mathbb{N}$ satisfies equation (14). Since $m=k+\left[m^{\theta}\right]-1 \geq k$ it also follows that

$$
m \geq k+\left[k^{\theta}\right]-1
$$


Next we claim that for each $\gamma>1$ there exists $\nu_{\gamma}>0$, depending only on $\gamma$ and $\theta$ such that

$$
m \leq k+\left[\gamma k^{\theta}\right]
$$

for all natural $k \geq \nu_{\gamma}$.

Indeed, equality (14) implies that for each $0<\delta<1$

$$
k \geq m-m^{\theta}=m\left(1-m^{\theta-1}\right) \geq \delta m \quad \text { if } m \geq(1-\delta)^{1 /(\theta-1)} .
$$

Hence for all natural $k \geq(1-\delta)^{1 /(\theta-1)}\left(\Rightarrow m \geq(1-\delta)^{1 /(\theta-1)}\right)$

$$
k \geq m-\left[m^{\theta}\right] \geq m-\left[\delta^{-\theta} k^{\theta}\right] \Rightarrow m \leq k+\left[\delta^{-\theta} k^{\theta}\right]=k+\left[\gamma k^{\theta}\right]
$$

if $\delta^{-\theta}=\gamma$. So inequality (15) holds for $k \geq \nu_{\gamma}=\left(1-\gamma^{-1 / \theta}\right)^{1 /(\theta-1)}$.

3. Inequalities (13) and (15) imply that for natural $k \geq \max \left\{k_{2}, \nu_{\gamma}\right\}$

$$
s_{k+\left[\gamma k^{\theta}\right]}(A) \leq s_{k}(A+B)\left(1+c_{4}\left(k+\left[\gamma k^{\theta}\right]\right)^{\alpha-\theta \beta}\right) \leq s_{k}(A+B)\left(1+c_{4} k^{\alpha-\theta \beta}\right) .
$$

Therefore

$$
s_{k}(A+B) \geq s_{k+\left[\gamma k^{\theta}\right]}(A)\left(1-c_{4} k^{\alpha-\theta \beta}\right) .
$$

If $k \geq\left(2 c_{4}\right)^{1 /(\theta \beta-\alpha)}$, then $1-c_{4} k^{\alpha-\theta \beta} \geq 1 / 2$.

Also by (10) for $k \geq 2$

$$
s_{k}(A+B) \leq s_{k-\left[k^{\theta}\right]}(A)\left(1+c_{4} k^{\alpha-\theta \beta}\right),
$$

since $c_{4}>c_{3}$.

Hence, the statement follows for

$$
k \geq \varkappa_{\gamma}=\left[\max \left\{k_{2}, \nu_{\gamma},\left(2 c_{4}\right)^{1 /(\theta \beta-\alpha)}, 2\right\}\right]+1 .
$$

Remark 3.2. Applying more delicate estimates one can prove that inequality (15) follows from the inequality $x \leq \gamma\left((x+1)^{1 / \theta}-x\right)^{\theta}, x \geq x_{\gamma}$, which implies that for $0<\theta \leq 1 / 2$ inequality (15) and hence inequality (12) also hold with $\gamma=1$.

Definition 3.1. Let $A$ and $B$ be compact linear operators in a Hilbert space $H$.

If $c_{1}, c_{2}>0,0<\alpha<\beta$, and condition (9) holds, we say that in the representation $C=A+B$ the operator $A$ is a leading operator and the operator $B$ is a non-leading operator with the parameters $c_{1}, c_{2}>0, \alpha$, and $\beta$.

If $0<\alpha<\beta$ and there exist $c_{1}, c_{2}>0$ such that condition (9) holds, we say that in the representation $C=A+B$ the operator $A$ is a leading operator and the operator $B$ is a non-leading operator with the parameters $\alpha$ and $\beta$.

If there exist $c_{1}, c_{2}>0$ and $0<\alpha<\beta$ such that condition (9) holds, we say that in the representation $C=A+B$ the operator $A$ is a leading operator and the operator $B$ is a non-leading operator.

Theorem 3.1. Let $A$ and $B$ be compact linear operators in a Hilbert space $H$. If in the representation $C=A+B$ the operator $A$ is a leading operator and the operator $B$ is a non-leading operator and for all $0<\sigma<1$

$$
\lim _{k \rightarrow \infty} \frac{s_{k+\left[k^{\sigma}\right]}(A)}{s_{k}(A)}=1
$$

then

$$
\lim _{k \rightarrow \infty} \frac{s_{k}(A+B)}{s_{k}(A)}=1 .
$$


Proof. Let $A$ be a leading operator and $B$ a non-leading operator with the parameters $\alpha, \beta$ and in Lemma 3.2 let $\gamma=2$ and $\theta=\frac{1}{2}\left(\frac{\alpha}{\beta}+1\right)$. Moreover, let $\sigma=\frac{1}{2}(\theta+1)$.

Given that $k \in \mathbb{N}$, let $m=k-\left[k^{\theta}\right]$. By the proof of Lemma 3.2 (see (14) and (15)) $k \leq m+1+\left[2(m+1)^{\theta}\right]$ for all $k \geq\left(1-2^{-1 / \theta}\right)^{1 /(\theta-1)}$.

Hence there exists $\varkappa \in \mathbb{N}$, depending only on $\alpha$ and $\beta$ such that for all $k \geq \varkappa$

$$
k+\left[2 k^{\theta}\right] \leq k+\left[k^{\sigma}\right], \quad k \leq m+\left[m^{\sigma}\right] .
$$

Therefore

$$
\frac{s_{k+\left[k^{\sigma}\right]}(A)}{s_{k}(A)} \leq \frac{s_{k+\left[2 k^{\theta}\right]}(A)}{s_{k}(A)} \leq 1 \leq \frac{s_{k-\left[k^{\sigma}\right]}(A)}{s_{k}(A)}=\frac{s_{m}(A)}{s_{k}(A)} \leq \frac{s_{m}(A)}{s_{m+\left[m^{\sigma}\right]}(A)} .
$$

Consequently

$$
\lim _{k \rightarrow \infty} \frac{s_{k+\left[2 k^{\theta}\right]}(A)}{s_{k}(A)}=\lim _{k \rightarrow \infty} \frac{s_{k-\left[k^{\theta}\right]}(A)}{s_{k}(A)}=1,
$$

and the statement follows by inequality (12).

Remark 3.3. Assume that, in addition to assumption (9)

$$
\lim _{k \rightarrow \infty} k^{\alpha} s_{k}(A)=a>0
$$

or, more generally,

$$
\lim _{k \rightarrow \infty} k^{\alpha} L(k) s_{k}(A)=a>0,
$$

where $L$ is an arbitrary positive function on $[1, \infty)$ for which for any $0<b<\infty$

$$
\lim _{k \rightarrow \infty} \frac{L(b k)}{L(k)}=1 \text {. }
$$

Then the statement of Theorem 3.1 follows from Theorem 2.3 in [8, Chapter 2, according to which if (18) or (19) is satisfied and

$$
\lim _{k \rightarrow \infty} \frac{s_{k}(B)}{s_{k}(A)}=0
$$

then equality (17) holds.

Corollary 3.1. Let $\mathfrak{A}, \mathfrak{B}$ be families of compact linear operators $A, B$ respectively, in a Hilbert space $H$. If there exist $c_{1}, c_{2}>0$ and $0<\alpha<\beta$ such that in the representations $C=A+B$ for all $A \in \mathfrak{A}, B \in \mathfrak{B}$ the operators $A$ are leading operators and the operators $B$ are non-leading operators with the parameters $c_{1}, c_{2}>0$ and $0<\alpha<\beta$ and, for all $0<\sigma<1$, in (16) convergence is uniform with respect to $A \in \mathfrak{A}$, then in (17) convergence is uniform with respect to $A \in \mathfrak{A}, B \in \mathfrak{B}$.

Proof. This proof follows from the proof of Theorem 3.1 and from Lemma 3.2, because the parameter $\varkappa$ in the above proof depends only on $\alpha$ and $\beta$, and the parameter $c_{4}$ in inequality (10) depends only on $c_{1}, c_{2}>0, \alpha$, and $\beta$.

\section{$\S$ 4. Some estimates For Singular numbers of ELliptic OPERATORS}

Let $l \in \mathbb{N}$ and for $u \in C^{\infty}\left(\mathbb{R}^{n}\right)$

$$
(\mathcal{L} u)(x)=\sum_{|\alpha|,|\beta| \leq l}(-1)^{|\alpha|+|\beta|} D^{\alpha}\left(A_{\alpha \beta}(x) D^{\beta} u\right), \quad x \in \mathbb{R}^{n},
$$

where $A_{\alpha \beta} \in C^{l}\left(\mathbb{R}^{n}\right)$ are real-valued functions for all multi-indices $\alpha, \beta$ satisfying $|\alpha|,|\beta| \leq l$. We assume the uniform ellipticity condition is satisfied, i. e. for some $\nu>0$

$$
\sum_{|\alpha|=|\beta|=l} A_{\alpha \beta}(x) \xi^{\alpha} \xi^{\beta} \geq \nu|\xi|^{2 l}
$$

for all $x, \xi \in \mathbb{R}^{n}$. 
We shall consider the closures of $\mathcal{L}$ in $L_{2}(\Omega)$ for bounded domains $\Omega \subseteq \mathbb{R}^{n}$ which will be denoted by $L_{\Omega}$.

Lemma 4.1. Let $\Omega \subseteq \mathbb{R}^{n}$ be a bounded domain.

1. The singular numbers $11 s_{k}(A)$ of any correct restriction $A$ of the operator $L_{\Omega}$ defined above whose inverse $A^{-1}$ is compact, satisfy the following inequality: there exists $c_{1}>0$ such that for all $k \in \mathbb{N}$

$$
s_{k}(A) \leq c_{1} k^{2 l / n}
$$

2. There exists a correct restriction $A$ of the operator $L_{\Omega}, k_{0} \in \mathbb{N}$, and $c_{2}, c_{3}>0$ such that for all $k \in \mathbb{N}, k \geq k_{0}$

$$
c_{2} k^{2 l / n} \leq s_{k}(A) \leq c_{3} k^{2 l / n} .
$$

Proof. 1. Let $B_{1} \subseteq \Omega$ be an open ball. Moreover, let $P_{1}: D\left(P_{1}\right) \rightarrow L_{2}(\Omega)$ be the operator generated by $L_{\Omega}$ with the homogeneous Dirichlet boundary conditions, i. e.

$$
D\left(P_{1}\right)=\left\{u \in W_{2}^{2 l}\left(B_{1}\right): \operatorname{tr}_{\partial B_{1}}\left(\frac{\partial^{m} f}{\partial n^{m}}\right)=0, m=0,1, \ldots, l-1\right\}
$$

and $P_{1} u=L_{\Omega} u$ for each $u \in D\left(P_{1}\right)$. It is well known that the operator $P_{1}$ has discrete spectrum and and its singular numbers satisfy the following inequality: there exist $k_{0} \in \mathbb{N}$ and $c_{4}, c_{5}>0$ such that for all $k \in \mathbb{N}, k \geq k_{0}$

$$
c_{4} k^{2 l / n} \leq s_{k}\left(P_{1}\right) \leq c_{5} k^{2 l / n}
$$

2. Let $T: W_{2}^{2 l}\left(B_{1}\right) \rightarrow W_{2}^{2 l}\left(\mathbb{R}^{n}\right)$ be a bounded linear extension operator. Then for any correct restriction $A$ of $L_{\Omega}$ and for any $u \in D\left(P_{1}\right)$

$$
\|A T u\|_{L_{2}(\Omega)} \leq c_{6}\|T u\|_{W_{2}^{2 l}(\Omega)} \leq c_{6} c_{7}\|u\|_{W_{2}^{2 l}\left(B_{1}\right)} \leq c_{6} c_{7} c_{8}\left\|P_{1} u\right\|_{L_{2}\left(B_{1}\right)} .
$$

Here

$$
c_{6}=\widetilde{c}_{6} \max _{|\alpha|,|\beta| \leq l} \max _{|\gamma| \leq l} \sup _{x \in \Omega}\left|\left(D^{\gamma} A_{\alpha \beta}\right)(x)\right|,
$$

where $\widetilde{c}_{6}$ depends only on $n$ and $l, c_{7}$ is the norm of the extension operator $T$, and $c_{8}$ is the norm of the inverse 2 operator $P_{1}^{-1}: L_{2}\left(B_{1}\right) \rightarrow W_{2}^{2 l}\left(B_{1}\right)$.

By the Min-Max principle it follows that for all $k \in \mathbb{N}$

$$
\begin{aligned}
s_{k}(A) & =\inf _{\substack{M \subseteq D(A) \\
\operatorname{dim} M=k}} \sup _{v \in M, v \nsim 0} \frac{\|A v\|_{L_{2}(\Omega)}}{\|v\|_{L_{2}(\Omega)}} \leq \inf _{\substack{M \subseteq D\left(P_{1}\right) \\
\operatorname{dim} M=k}} \sup _{u \in M, u \nsim 0} \frac{\|A T u\|_{L_{2}(\Omega)}}{\|T u\|_{L_{2}(\Omega)}} \\
\leq & c_{6} c_{7} c_{8} \inf _{\substack{M \subseteq D\left(P_{1}\right) \\
\operatorname{dim} M=k}} \sup _{u \in M, u \nsim 0} \frac{\left\|P_{1} u\right\|_{L_{2}\left(B_{1}\right)}}{\|u\|_{L_{2}\left(B_{1}\right)}}=c_{6} c_{7} c_{8} s_{k}\left(P_{1}\right) \leq c_{5} c_{6} c_{7} c_{8} k^{2 l / n} .
\end{aligned}
$$

3. Let $B_{2}$ be an open ball such that $\Omega \subseteq B_{2}$ and let $P_{2}$ be the operator similar to $P_{1}$ but defined on $B_{2}$. Moreover, let $T_{0}: L_{2}(\Omega) \rightarrow L_{2}\left(B_{2}\right)$ be the extension-by-zero operator and $R_{\Omega}: L_{2}\left(B_{2}\right) \rightarrow L_{2}(\Omega)$ be the restriction operator. We define

$$
B=R_{\Omega} P_{2}^{-1} T_{0}
$$

Then $B$ is a bounded linear operator for which

$$
L_{\Omega} B=L_{\Omega} R_{\Omega} P_{2}^{-1} T_{0}=R_{\Omega} P_{2} P_{2}^{-1} T_{0}=R_{\Omega} T_{0}=I
$$

\footnotetext{
${ }^{1}$ As usual it is assumed that all singular numbers of $A$ are written in the form of a sequence $\left\{s_{k}(A)\right\}_{k=1}^{\infty}$, where $s_{1}(A) \leq s_{2}(A) \leq \cdots \leq s_{k}(A) \leq \ldots$ and each singular number is repeated as many times as its multiplicity.

${ }^{2}$ Here and in similar situations in the sequel we assume, without loss of generality, that 0 does not belong to the spectrum of $P_{1}$. Otherwise, we may consider the operator $P_{1}+\mu I$ with the appropriately chosen $\mu \in \mathbb{R}$.
} 
and

$$
R(B) \subseteq R_{\Omega}\left(D\left(P_{2}\right)\right) \subseteq W_{2}^{2 l}(\Omega) \subseteq D\left(L_{\Omega}\right)
$$

By Remark $2.1 B$ is the inverse of a certain correct restriction $A$ of the operator $L_{\Omega}$. So

$$
A^{-1}=R_{\Omega} P_{2}^{-1} T_{0}
$$

This implies that there exist $c_{9}, c_{10}>0$ such that $c_{9} s_{k}\left(P_{2}\right) \leq s_{k}(A) \leq c_{10} s_{k}\left(P_{2}\right)$ for all $k \in \mathbb{N}$. Hence, by (24), where the operator $P_{1}$ and the ball $B_{1}$ are replaced by $P_{2}$ and $B_{2}$, the statement follows.

Remark 4.1. Let $\Omega$ contain an open ball $B(x, r)$ of radius $r>0$ for some $x \in \Omega$ and be contained in the ball $B(0, R)$. Moreover, let

$$
\tau=\max _{|\alpha|,|\beta| \leq l} \max _{|\gamma| \leq l} \sup _{x \in B(0, R)}\left|\left(D^{\gamma} A_{\alpha, \beta}\right)(x)\right| .
$$

Then the above proof implies that it may be assumed that in inequality (22) $c_{1}$ depends only on $n, l, r, R, \tau$, and the ellipticity constant $\nu$.

\section{$\S 5$. Classes of domains}

For any set $V$ in $\mathbb{R}^{n}$ and $\delta>0$ we denote by $V_{\delta}$ the set $\{x \in V: d(x, \partial \Omega)>\delta\}$ and by $V^{\delta}$ the $\delta$-neighbourhood of $V$.

Let $n \in \mathbb{N}, n \geq 2, \rho>0, s, s^{\prime} \in \mathbb{N}, s^{\prime} \leq s$ and $\left\{V_{j}\right\}_{j=1}^{s}$ be a family of bounded open cuboids and $\left\{r_{j}\right\}_{j=1}^{s}$ be a family of rotations in $\mathbb{R}^{n}$.

We say that that $\mathcal{A}=\left(\rho, s, s^{\prime},\left\{V_{j}\right\}_{j=1}^{s},\left\{r_{j}\right\}_{j=1}^{s}\right)$ is an atlas in $\mathbb{R}^{n}$ with the parameters $\rho, s, s^{\prime},\left\{V_{j}\right\}_{j=1}^{s},\left\{r_{j}\right\}_{j=1}^{s}$, briefly an atlas in $\mathbb{R}^{n}$.

Let $l \in \mathbb{N}$ and $M>0$. We denote by $C_{M}^{l}(\mathcal{A})$ the family of all bounded domains $\Omega$ in $\mathbb{R}^{n}$ satisfying the following properties:

(i) $\Omega \subseteq \bigcup_{j=1}^{s}\left(V_{j}\right)_{\rho}$ and $\left(V_{j}\right)_{\rho} \cap \Omega \neq \emptyset$;

(ii) $V_{j} \cap \partial \Omega \neq \emptyset$ for $j=1, \ldots, s^{\prime}, V_{j} \cap \partial \Omega=\emptyset$ for $s^{\prime}<j \leq s$;

(iii) for $j=1, \ldots, s$

$$
r_{j}\left(V_{j}\right)=\left\{x \in \mathbb{R}^{n}: a_{i j}<x_{i}<b_{i j}, i=1, \ldots, n\right\}
$$

and

$$
r_{j}\left(\Omega \cap V_{j}\right)=\left\{x \in \mathbb{R}^{n}: a_{n j}<x_{n}<g_{j}(\bar{x}), \bar{x} \in W_{j}\right\},
$$

where $\bar{x}=\left(x_{1}, \ldots, x_{n-1}\right), W_{j}=\left\{\bar{x} \in \mathbb{R}^{n-1}: a_{i j}<x_{i}<b_{i j}, i=1, \ldots, n-1\right\}$ and $g_{j} \in C^{l}\left(\bar{W}_{j}\right)$ (it is meant that if $s^{\prime}<j \leq s$, then $g_{j}(\bar{x})=b_{n j}$ for all $\bar{x} \in \bar{W}_{j}$ ); moreover, for $j=1, \ldots, s^{\prime}$

$$
a_{n j}+\rho \leq g_{j}(\bar{x}) \leq b_{n j}-\rho
$$

and

$$
\left|D^{\alpha} g_{j}(\bar{x})\right| \leq M, \quad 1 \leq|\alpha| \leq l
$$

for all $\bar{x} \in \bar{W}_{j}$.

We say that a domain $\Omega$ in $\mathbb{R}^{n}$ is of class $C^{l}$ (or with boundary of class $C^{l}$ ) if $\Omega \in$ $C_{M}^{l}(\mathcal{A})$ for some atlas $\mathcal{A}$ and $M>0$. 


\section{$\S$ 6. RePRESEntation of the INVERSE OF A CORRECT RESTRICTION AS THE SUM OF A LEADING AND A NON-LEADING OPERATOR}

In this section we prove the core result of the paper.

Theorem 6.1. Let $l, n \in \mathbb{N}, n \geq 2$ and $\Omega$ be a bounded domain in $\mathbb{R}^{n}$ with the boundary $\partial \Omega$ of class $C^{2 l}$.

Let $A$ and $B$ be correct restrictions of the operator $L_{\Omega}$, defined in Section 4 , such that

$$
\begin{aligned}
& D(A) \subseteq W_{2}^{2 l}(\Omega), \\
& D(B) \subseteq W_{2}^{s}(\Omega),
\end{aligned}
$$

wher $3^{3}$

$$
2 l\left(1-\frac{1}{n}\right)<s \leq 2 l,
$$

and the operators

$$
\begin{aligned}
& A^{-1}: L_{2}(\Omega) \rightarrow W_{2}^{2 l}(\Omega), \\
& B^{-1}: L_{2}(\Omega) \rightarrow W_{2}^{s}(\Omega)
\end{aligned}
$$

are bounded.

Then in the representation

$$
B^{-1}=A^{-1}+K
$$

the operator $A^{-1}$ is a leading operator and the operator $K=B^{-1}-A^{-1}$ is a non-leading operator with the parameters $\alpha=2 l / n$ and any $2 l / n<\beta<s /(n-1)$.

Proof. 1. We start by stating several corollaries of the general results on the solvability of the boundary value problems proved in [10. Here we use the definitions of Sobolev spaces $W_{2}^{r}(\partial \Omega)$ for arbitrary real $r$ given in that book.

Let

$$
B_{m} u=\operatorname{tr}_{\partial \Omega}\left(\frac{\partial^{m} u}{\partial n^{m}}\right), \quad m=0,1, \ldots, l-1
$$

and $0<\sigma \leq 2 l$. Then the following Dirichlet problem

$$
\left\{\begin{array}{l}
u \in W_{2}^{\sigma}(\Omega) \\
L_{\Omega} u=0 \text { on } \Omega \\
B_{m} u=h_{m} \text { on } \partial \Omega, m=0,1, \ldots, l-1, \\
h_{m} \in W_{2}^{\sigma-m-1 / 2}(\partial \Omega)
\end{array}\right.
$$

has a unique solution 4 . Moreover, there exists $c_{1}>0$ such that

$$
\|u\|_{W_{2}^{\sigma}(\Omega)} \leq c_{1} \sum_{m=0}^{l-1}\left\|h_{m}\right\|_{W_{2}^{\sigma-m-1 / 2}(\partial \Omega)}
$$

for all collections of functions $h_{m} \in W_{2}^{\sigma-m-1 / 2}(\partial \Omega), m=0,1, \ldots, l-1$. Note that, for sufficiently small positive $\sigma, \sigma-m-1 / 2<0$.

${ }^{3}$ For $s>0, s \notin \mathbb{N}, W_{2}^{s}(\Omega)$ is the Sobolev space of fractional order with the norm

$$
\|u\|_{W_{2}^{s}(\Omega)}=\left(\sum_{|\alpha| \leq[s]} \int_{\Omega}\left|D_{w}^{\alpha} u(x)\right|^{2} d x+\sum_{|\alpha|=[s]} \int_{\Omega} \int_{\Omega} \frac{\left|D_{w}^{\alpha} u(x)-D_{w}^{\alpha} u(y)\right|^{2}}{|x-y|^{n+2(s-[s])}} d x d y\right)^{1 / 2},
$$

where $D_{w}^{\alpha} u$ denotes the weak derivative of $u$ of order $\alpha$ on $\Omega$.

${ }^{4} \mathrm{We}$ assume that 0 does not belong to the spectrum of the corresponding Dirichlet operator. See footnote 2 . 
Assume that $0<\mu<s$. We shall use the above statement for $\sigma=s$ and $\sigma=\mu$. If $\sigma=\mu$ this implies, in particular, that for each $j=0,1, \ldots, l-1$ the Dirichlet problem

$$
\left\{\begin{array}{l}
u \in W_{2}^{\mu}(\Omega) \\
L_{\Omega} u=0 \text { on } \Omega \\
B_{m} u=0 \text { on } \partial \Omega, m=0,1, \ldots, j-1, j+1, \ldots, l-1, \\
B_{j} u=h \text { on } \partial \Omega \\
h \in W_{2}^{\mu-j-1 / 2}(\partial \Omega)
\end{array}\right.
$$

has a unique solution and the corresponding inverse operator

$$
R_{j}: W_{2}^{\mu-j-1 / 2}(\partial \Omega) \rightarrow W_{2}^{\mu}(\Omega)
$$

is bounded.

Note that the solution of the problem (33) with $\sigma=\mu$ can be represented in the form

$$
u=\sum_{j=0}^{l-1} R_{j} h_{j} .
$$

Since for each function $f \in L_{2}(\Omega)$ by (30) and (31) we have $K f=B^{-1} f-A^{-1} f \in$ $W_{2}^{s}(\Omega), L_{\Omega} K f=0$ on $\Omega$ and by the trace theorem for Sobolev spaces (note that by (29) $s>l-1 / 2)$

$$
B_{m} K f \in W_{2}^{s-m-1 / 2}(\partial \Omega) \subseteq W_{2}^{\mu-m-1 / 2}(\partial \Omega), \quad m=0,1, \ldots, l-1,
$$

this implies that

$$
K f=\sum_{j=0}^{l-1} R_{j} B_{j} K f, \quad f \in L_{2}(\Omega) .
$$

2. We shall make use of the following fact: for a compact linear operator $G: H_{1} \rightarrow H_{2}$ acting from a Hilbert space $H_{1}$ to a Hilbert space $H_{2}$,

$$
s_{1}(G)=\|G\| ; \quad s_{k}(G)=\min _{\operatorname{dim} F=k-1}\|A-F\|, \quad k \geq 2,
$$

where the minimum is taken with respect to all linear operators $F: H_{1} \rightarrow H_{2}$ of dimension $k-1$. (See [1] and [8, Theorem 2.1].)

3. Consider the embedding operator

$$
E_{1}: W_{2}^{\mu}(\Omega) \rightarrow L_{2}(\Omega) .
$$

This operator is compact and its singular numbers $s_{k}\left(E_{1}\right)$ have the order $k^{-\mu / n}$. Hence, there exist linear operators $M_{k}: W_{2}^{\mu}(\Omega) \rightarrow L_{2}(\Omega)$ of dimension $k, k \in \mathbb{N}$, such that

$$
\left\|\left(E_{1}-M_{k}\right) g\right\|_{L_{2}(\Omega)}=s_{k+1}\left(E_{1}\right) \leq c_{2}(k+1)^{-\mu / n}\|g\|_{W_{2}^{\mu}(\Omega)},
$$

where $c_{2}>0$ is independent of $k$ and $g \in W_{2}^{\mu}(\Omega)$.

Consider also the embedding operators

$$
E_{2 j}: W_{2}^{s-j-1 / 2}(\partial \Omega) \rightarrow W_{2}^{\mu-j-1 / 2}(\partial \Omega), \quad j=0,1, \ldots, l-1 .
$$

These operators are compact and their singular numbers $s_{k}\left(E_{2 j}\right)$ have the same order $k^{-(s-\mu) /(n-1)}$. (See [12].) Hence, there exist linear operators

$$
F_{k j}: W_{2}^{s-j-1 / 2}(\partial \Omega) \rightarrow W_{2}^{\mu-j-1 / 2}(\partial \Omega)
$$

of dimension $k, k \in \mathbb{N}$, such that

(38) $\left\|\left(E_{2 j}-F_{k j}\right) g\right\|_{W_{2}^{s-j-1 / 2}(\partial \Omega)}=s_{k+1}\left(E_{2 j}\right) \leq c_{3}(k+1)^{-(s-\mu) /(n-1)}\|g\|_{W_{2}^{\mu-j-1 / 2}(\partial \Omega)}$, 
where $c_{3}>0$ is independent of $j, k$ and $g \in W_{2}^{\mu-j-1 / 2}(\partial \Omega)$.

4. Denote by $T_{k}, k \in \mathbb{N}$, the operators defined by

$$
T_{k} f=K f-\sum_{j=0}^{l-1}\left(E_{1}-M_{k}\right) R_{j}\left(E_{2 j}-F_{k j}\right) B_{j} K f, \quad f \in L_{2}(\Omega) .
$$

By (36)

$$
\sum_{j=0}^{l-1} E_{1} R_{j} E_{2 j} B_{j} K f=\sum_{j=0}^{l-1} R_{j} B_{j} K f=K f .
$$

Therefore

$$
T_{k} f=\sum_{j=0}^{l-1} E_{1} R_{j} F_{k j} B_{j} K f+M_{k}\left(\sum_{j=0}^{l-1} R_{j}\left(E_{2 j}-F_{k j}\right) B_{j} K f\right) .
$$

Hence, $T_{k}: L_{2}(\Omega) \rightarrow L_{2}(\Omega)$ are linear operators of dimension not exceeding $(l+1) k$.

Moreover, by (37)

$$
\begin{aligned}
\left\|\left(K-T_{k}\right) f\right\|_{L_{2}(\Omega)} & =\left\|\sum_{j=0}^{l-1}\left(E_{1}-M_{k}\right) R_{j}\left(E_{2 j}-F_{k j}\right) B_{j} K f\right\|_{L_{2}(\Omega)} \\
& \leq \sum_{j=0}^{l-1}\left\|\left(E_{1}-M_{k}\right) R_{j}\left(E_{2 j}-F_{k j}\right) B_{j} K f\right\|_{L_{2}(\Omega)} \\
& \leq c_{2}(k+1)^{-\mu / n} \sum_{j=0}^{l-1}\left\|R_{j}\left(E_{2 j}-F_{k j}\right) B_{j} K f\right\|_{W_{2}^{\mu}(\Omega)} \\
& \leq c_{2} c_{4}(k+1)^{-\mu / n} \sum_{j=0}^{l-1}\left\|\left(E_{2 j}-F_{k j}\right) B_{j} K f\right\|_{W_{2}^{\mu-j-1 / 2}(\partial \Omega)},
\end{aligned}
$$

where

$$
c_{4}=\max _{j=0, \ldots, l-1}\left\|R_{j}\right\|_{W_{2}^{\mu-j-1 / 2}(\partial \Omega) \rightarrow W_{2}^{\mu}(\Omega)} .
$$

Next, by (38) and the trace theorem for Sobolev spaces

$$
\begin{aligned}
& \left\|\left(K-T_{k}\right) f\right\|_{L_{2}(\Omega)} \leq c_{2} c_{3} c_{4}(k+1)^{-\mu / n-(s-\mu) /(n-1)} \sum_{j=0}^{l-1}\left\|B_{j} K f\right\|_{W_{2}^{s-j-1 / 2}(\partial \Omega)} \\
& \leq c_{2} c_{3} c_{4} c_{5}(k+1)^{-\frac{1}{n-1}(s-\mu / n)}\|K f\|_{W_{2}^{s}(\Omega)} \leq c_{2} c_{3} c_{4} c_{5} c_{6}(k+1)^{-\frac{1}{n-1}(s-\mu / n)}\|f\|_{L_{2}(\Omega)},
\end{aligned}
$$

where

$$
\begin{aligned}
& c_{5}=\sum_{j=0}^{l-1}\left\|B_{j}\right\|_{W_{2}^{s}(\Omega) \rightarrow W_{2}^{s-j-1 / 2}(\partial \Omega)}, \\
& \begin{aligned}
c_{6}=\|K\|_{L_{2}(\Omega) \rightarrow W_{2}^{s}(\Omega)} & \leq\left\|B^{-1}\right\|_{L_{2}(\Omega) \rightarrow W_{2}^{s}(\Omega)}+\left\|A^{-1}\right\|_{L_{2}(\Omega) \rightarrow W_{2}^{s}(\Omega)} \\
& \leq\left\|B^{-1}\right\|_{L_{2}(\Omega) \rightarrow W_{2}^{s}(\Omega)}+c_{7}\left\|A^{-1}\right\|_{L_{2}(\Omega) \rightarrow W_{2}^{2 l}(\Omega)}
\end{aligned}
\end{aligned}
$$

and $c_{7}$ is the norm of the embedding operator $E: W_{2}^{2 l}(\Omega) \rightarrow W_{2}^{s}(\Omega)$.

5. Given $\frac{2 l}{n}<\beta<\frac{s}{n-1}$, we choose $0<\mu<s$ such that $\frac{1}{n-1}\left(s-\frac{\mu}{n}\right)=\beta$. This is possible if and only if $s>2 l\left(1-\frac{1}{n}\right)$. So for all $\frac{2 l}{n}<\beta<\frac{s}{n-1}$

$$
\left\|\left(K-T_{k}\right) f\right\|_{L_{2}(\Omega)} \leq c_{8}(k+1)^{-\beta}\|f\|_{L_{2}(\Omega)},
$$


where

$$
c_{8}=c_{2} c_{3} c_{4} c_{5}\left(\left\|B^{-1}\right\|_{L_{2}(\Omega) \rightarrow W_{2}^{s}(\Omega)}+c_{7}\left\|A^{-1}\right\|_{L_{2}(\Omega) \rightarrow W_{2}^{2 l}(\Omega)}\right)
$$

is independent of $k \in \mathbb{N}$ and $f \in L_{2}(\Omega)$.

Since $\operatorname{dim} T_{\left[\frac{k-1}{l+1}\right]} \leq k-1$ we have

$$
\begin{aligned}
s_{k}(K) \leq\left\|K-T_{\left[\frac{k-1}{l+1}\right]}\right\|_{L_{2}(\Omega) \rightarrow L_{2}(\Omega)} & \leq c_{8}\left(\left[\frac{k-1}{l+1}\right]+1\right)^{-\beta} \\
& \leq c_{8}(l+1)^{\beta}(k-1)^{\beta} \leq c_{8}(2(l+1))^{\beta} k^{-\beta}
\end{aligned}
$$

for all $k \geq 2$. This inequality also holds for $k=1$ because without loss of generality one may assume that $c_{2}, c_{3}, c_{4}, c_{5} \geq 1$, in which case $s_{1}(K)=\|K\| \leq c_{8}$.

Finally, by Lemma 4.1 it follows that for all $k \in \mathbb{N}$

$$
s_{k}\left(A^{-1}\right) \geq c_{1}^{-1} k^{-2 l / n}
$$

where $c_{1}$ is from inequality (22), which implies the statement.

Analysis of the proof. Estimate (37) with $\mu=s$ implies immediately that for $k \geq 2$

$$
\begin{aligned}
s_{k}(K) & \leq\left\|K-M_{k-1} K\right\|_{L_{2}(\Omega) \rightarrow L_{2}(\Omega)}= \\
& =\left\|\left(E_{1}-M_{k-1}\right) K\right\|_{L_{2}(\Omega) \rightarrow L_{2}(\Omega)} \leq c_{2}\|K\|_{W_{2}^{s}(\Omega) \rightarrow L_{2}(\Omega)} k^{-s / n} .
\end{aligned}
$$

This is a rather rough estimate and combined with estimate (42) it does not allow us to claim that $K$ is a non-leading operator. However, this estimate holds for any bounded linear operator $K: W_{2}^{s}(\Omega) \rightarrow L_{2}(\Omega)$ whilst the operator $K=B^{-1}-A^{-1}$ under consideration apart from that is such that $L_{\Omega} K=0$. This essential fact enables us to improve significantly estimate (43). In the above proof this is done by considering $K f$ as a solution of the Dirichlet boundary value problem for the operator $L_{\Omega}$ with the boundary data $B_{m} K f \in W_{2}^{s-m-1 / 2}(\partial \Omega), m=0, \ldots, l-1$, where $s-m-1 / 2>0$, using estimate (38) with any $0<\mu<s$ and deep results from [10] on solvability of this Dirichlet problem for the case in which the boundary data belongs to Sobolev spaces of negative order.

Example 6.1. In Example 2.2 let $\varphi_{1} \in W_{2}^{s}(B)$ where $2-2 / n<s \leq 2$. Consider the operator $L_{s}: D\left(L_{s}\right) \rightarrow L_{2}(B)$ where

$$
D\left(L_{s}\right)=\left\{u \in L_{2}(B):-\Delta_{w} u \in L_{2}(B), \operatorname{tr}_{\partial B}\left(u(x)+\varphi_{1}(x) \int_{B} \varphi_{2}(y) \Delta_{w} u(y) d y\right)=0\right\}
$$

and $L_{s} u=-\Delta_{w} u$ for $u \in D\left(L_{s}\right)$. By Examples 2.1 and $2.2 L_{s}$ is a correct restriction of the operator $L$. Moreover, the operator $L_{s}^{-1}: L_{2}(B) \rightarrow W_{2}^{s}(B)$ is bounded. Therefore by Theorem 6.1 the singular numbers $s_{k}\left(L_{s}\right)$ have the same asymptotics as those of the Dirichlet Laplacian:

$$
\lim _{k \rightarrow \infty} s_{k}\left(L_{s}\right) k^{-2 / n}=\lim _{k \rightarrow \infty} s_{k}\left(-\Delta_{\mathrm{D}}\right) k^{-2 / n} .
$$

Corollary 6.1. Let $l, n \in \mathbb{N}, n \geq 2,2 l\left(1-\frac{1}{n}\right)<s \leq 2 l, \alpha=\frac{2 l}{n}$, and $\frac{2 l}{n}<\beta<\frac{s}{n-1}$. Moreover, let $\mathcal{A}$ be a fixed atlas in $\mathbb{R}^{n}$ and $M>0$.

Assume that $G, H \subseteq C_{M}^{2 l}(\mathcal{A})$ and $\mathfrak{A}(\mathcal{A})=\left\{A_{\Omega}\right\}_{\Omega \in G}, \mathfrak{B}(\mathcal{A})=\left\{B_{\Omega}\right\}_{\Omega \in H}$ are families of correct restrictions $A_{\Omega}$ and $B_{\Omega}$ of the operator $L_{\Omega}$ defined in Section 4, satisfying the conditions

$$
\begin{array}{ll}
D\left(A_{\Omega}\right) \subseteq W_{2}^{2 l}(\Omega), & A_{\Omega} \in \mathfrak{A}(\mathcal{A}), \\
D\left(B_{\Omega}\right) \subseteq W_{2}^{s}(\Omega), & B_{\Omega} \in \mathfrak{B}(\mathcal{A}),
\end{array}
$$


and

$$
\begin{aligned}
\theta_{\mathfrak{A}(\mathcal{A})} & =\sup _{A_{\Omega} \in \mathfrak{A}(\mathcal{A})}\left\|A_{\Omega}^{-1}\right\|_{L_{2}(\Omega) \rightarrow W_{2}^{2 l}(\Omega)}<\infty, \\
\theta_{\mathfrak{B}(\mathcal{A})} & =\sup _{B_{\Omega} \in \mathfrak{B}(\mathcal{A})}\left\|B_{\Omega}^{-1}\right\|_{L_{2}(\Omega) \rightarrow W_{2}^{s}(\Omega)}<\infty .
\end{aligned}
$$

Then there exist $c_{9}, c_{10}>0$ such that for all $A_{\Omega} \in \mathfrak{A}(\mathcal{A}), B_{\Omega} \in \mathfrak{B}(\mathcal{A})$ in the representations

$$
B_{\Omega}^{-1}=A_{\Omega}^{-1}+K_{\Omega}
$$

the operators $A_{\Omega}^{-1}$ are leading and the operators $K_{\Omega}$ are non-leading with the parameters $c_{9}, c_{10}, \alpha$, and $\beta$.

Proof. By Remark 4.1 it follows that there exists $c_{9}>0$, depending only on $n, l$, the operator $\mathcal{L}$, the atlas $\mathcal{A}$, and $M$ such that

$$
s_{k}\left(A_{\Omega}^{-1}\right)=s_{k}\left(A_{\Omega}\right)^{-1} \geq c_{9} k^{-2 l / n}
$$

for all $k \in \mathbb{N}$ and for all $A_{\Omega} \in \mathfrak{A}(\mathcal{A})$.

Tracing the proofs in [2], [10, and [12] and it follows that in the proof of Theorem 6.1 it may be assumed that $c_{2}, c_{3}, c_{5}, c_{7}$ depend only of $n, l, s, \beta$, the atlas $\mathcal{A}$, and $M$. As for $c_{1}$ and $c_{4}$, they also depend on the operator $\mathcal{L}$. Hence, by (40), (41), (46), and (47),

$$
s_{k}(K) \leq c_{10} k^{-\beta},
$$

where

$$
c_{10}=c_{2} c_{3} c_{4} c_{5}\left(\theta_{\mathfrak{B}(\mathcal{A})}+c_{7} \theta_{\mathfrak{A}(\mathcal{A})}\right)(2(l+1))^{\beta}
$$

for all $k \in \mathbb{N}$ and for all $A_{\Omega} \in \mathfrak{A}(\mathcal{A})$ and $B_{\Omega} \in \mathfrak{B}(\mathcal{A})$.

\section{$\S 7$. COMPARISON OF THE ASYMPTOTICS OF SINGUlAR NUMBERS OF CORRECT RESTRICTIONS OF ELLIPTIC OPERATORS}

Theorem 7.1. Let $l, n \in \mathbb{N}, n \geq 2,2 l(1-1 / n)<s \leq 2 l$ and $\Omega$ be a bounded domain in $\mathbb{R}^{n}$ with the boundary $\partial \Omega$ of class $C^{2 l}$.

Then there exists $b>0$ such that for all correct restrictions $B$ of the operator $L_{\Omega}$, defined in Section 4, satisfying conditions (28) and (31)

$$
\lim _{k \rightarrow \infty} s_{k}(B) k^{-2 l / n}=b \text {. }
$$

Proof. Let the correct restriction $A$ of the operator $L_{\Omega}$ be defined by

$$
D(A)=\left\{u \in W_{2}^{2 l}(\Omega): \operatorname{tr}_{\partial \Omega}\left(\frac{\partial^{m} u}{\partial n^{m}}\right)=0, m=0,1, \ldots, l-1\right\}
$$

and $A u=L_{\Omega} u$ for each $u \in D(A)$. Without loss of generality we assume that the operator $A$ has compact inverse $A^{-1}$ (see footnote 2 ). It is well known that its singular numbers $s_{k}\left(A^{-1}\right)$ are such that

for some $a>0$.

$$
\lim _{k \rightarrow \infty} s_{k}\left(A^{-1}\right) k^{2 l / n}=a
$$

Let $B$ be any correct restriction of the operator $L_{\Omega}$ satisfying conditions (28) and (31). By Theorem 6.1 $B^{-1}=A^{-1}+K$, where $A^{-1}$ is a leading operator and $K$ is a non-leading operator. Since condition (16) is satisfied by Theorem 3.1

$$
\lim _{k \rightarrow \infty} \frac{s_{k}\left(B^{-1}\right)}{s_{k}\left(A^{-1}\right)}=1 .
$$

Consequently

$$
\lim _{k \rightarrow \infty} s_{k}\left(B^{-1}\right) k^{2 l / n}=\lim _{k \rightarrow \infty} s_{k}\left(A^{-1}\right) k^{2 l / n}=a
$$


and

$$
\lim _{k \rightarrow \infty} s_{k}(B) k^{-2 l / n}=b \equiv \frac{1}{a} .
$$

Corollary 7.1. Let $l, n \in \mathbb{N}, n \geq 2,2 l(1-1 / n)<s \leq 2 l$, $\mathcal{A}$ be a fixed atlas in $\mathbb{R}^{n}$, and $M>0$. Assume that $H \subseteq C_{M}^{2 l}(\mathcal{A})$ and $\mathfrak{B}(\mathcal{A})=\left\{B_{\Omega}\right\}_{\Omega \in H}$ is a family of correct restrictions $B_{\Omega}$ of the operator $L_{\Omega}$ defined in Section 4, satisfying conditions (28) and (47).

Then the expressions $s_{k}\left(B_{\Omega}\right) k^{-2 l / n}$ converge to $b$ as $k \rightarrow \infty$ uniformly with respect to $B_{\Omega} \in \mathfrak{B}(\mathcal{A})$.

Proof. Let the family $\mathfrak{A}(\mathcal{A})$ consist of only one operator $A$ defined above. Since condition (16) is satisfied the statement follows by Corollaries 3.1 and 6.1

\section{$\S 8$. Application to spectral stability estimates}

In this section we restrict ourselves to considering the operator $\mathcal{L}$ for the case in which $l=1, A_{\alpha \beta}=A_{\beta \alpha}$ and there are no lower terms. So for $u \in C^{\infty}\left(\mathbb{R}^{n}\right)$

$$
\mathcal{L} u=-\sum_{i, j=1}^{n} \frac{\partial}{\partial x_{i}}\left(a_{i j}(x) \frac{\partial u}{\partial x_{j}}\right), \quad x \in \mathbb{R}^{n},
$$

where $a_{i j} \in C^{1}\left(\mathbb{R}^{n}\right)$ are real-valued functions satisfying $a_{i j}=a_{j i} \forall i, j=1, \ldots, n$, and

$$
\sum_{i, j=1}^{n} a_{i j}(x) \xi_{i} \xi_{j} \geq \nu|\xi|^{2}
$$

for all $\xi, x \in \mathbb{R}^{n}$. As in previous sections, for bounded domains $\Omega \subseteq \mathbb{R}^{n}, L_{\Omega}$ denotes the closure of $\mathcal{L}$ in $L_{2}(\Omega)$.

Theorem 8.1. Let $n \in \mathbb{N}, n \geq 2,2-2 / n<s \leq 2, \mathcal{A}$ be a fixed atlas in $\mathbb{R}^{n}$, and $M>0$.

Moreover, let $\mathfrak{B}(\mathcal{A})=\left\{B_{\Omega}\right\}_{\Omega \in C_{M}^{2}(\mathcal{A})}$ be a family of correct restrictions $B_{\Omega}$ of the operator $L_{\Omega}$ defined above, satisfying conditions (28) and (47).

Then there exist $\varepsilon_{1}, c_{1}>0$ and for each $\varepsilon \in\left(0, \varepsilon_{1}\right]$ there exists $k(\varepsilon) \in \mathbb{N}$ such that for all $k \geq k(\varepsilon)$

$$
\left|s_{k}\left(B_{\Omega_{1}}\right)-s_{k}\left(B_{\Omega_{2}}\right)\right| \leq c_{1} k^{2 / n} \varepsilon
$$

for all $\Omega_{1}, \Omega_{2} \in C_{M}^{2}(\mathcal{A})$ satisfying

$$
\left(\Omega_{1}\right)_{\varepsilon} \subseteq \Omega_{2} \subseteq\left(\Omega_{1}\right)^{\varepsilon} \quad \text { or } \quad\left(\Omega_{2}\right)_{\varepsilon} \subseteq \Omega_{1} \subseteq\left(\Omega_{2}\right)^{\varepsilon} .
$$

Proof. 1. Let $\mathfrak{A}(\mathcal{A})=\left\{A_{\Omega}\right\}_{\Omega \in C_{M}^{2}(\mathcal{A})}$ be the family of the correct restrictions $A_{\Omega}$ of the operator $L_{\Omega}$ defined by

$$
D\left(A_{\Omega}\right)=\left\{u \in W_{2}^{2}(\Omega): \operatorname{tr}_{\partial \Omega} u=0\right\}
$$

and $A_{\Omega} u=L_{\Omega} u$ for each $u \in D\left(A_{\Omega}\right)$. By Theorem 7.15 in 3 there exist $\varepsilon_{2}, c_{2}>0$, depending only on $\mathcal{A}$ and $L$, such that the eigenvalues $\lambda_{k}\left(A_{\Omega}\right)$ satisfy the following estimate: for all $k \in \mathbb{N}$ for all $\varepsilon \in\left(0, \varepsilon_{2}\right]$

$$
\left|\lambda_{k}\left(A_{\Omega_{1}}\right)-\lambda_{k}\left(A_{\Omega_{2}}\right)\right| \leq c_{2} \lambda_{k}\left(A_{\Omega_{1}}\right) \varepsilon
$$

for all $\Omega_{1}, \Omega_{2} \in C_{M}^{2}(\mathcal{A})$ satisfying condition (50).

Note also that there exists $c_{3}>0$, depending only on $n, l, \mathcal{A}$ and $L$, such that for all $k \in \mathbb{N}$

$$
\lambda_{k}\left(A_{\Omega}\right) \leq c_{3} k^{2 / n}
$$

for all $A_{\Omega} \in \mathfrak{A}(\mathcal{A})$. 
This follows from Remark 4.1 since each $\Omega \in C_{M}^{2}(\mathcal{A})$ contains an open ball $B(x, \varrho / 2)$ for some $x \in \Omega$ and is contained in the ball $B(0, R)$, where $R>0$ depends only on $\left\{V_{j}\right\}_{j=1}^{s}$.

2. Next for all $k \in \mathbb{N}$ and for all $\Omega_{1}, \Omega_{2} \in C_{M}^{2}(\mathcal{A})$

$$
\begin{aligned}
\left|s_{k}\left(B_{\Omega_{1}}\right)-s_{k}\left(B_{\Omega_{2}}\right)\right| & \leq\left|s_{k}\left(B_{\Omega_{1}}\right)-\lambda_{k}\left(A_{\Omega_{1}}\right)\right|+\left|\lambda_{k}\left(A_{\Omega_{1}}\right)-\lambda_{k}\left(A_{\Omega_{2}}\right)\right|+\left|s_{k}\left(B_{\Omega_{2}}\right)-\lambda_{k}\left(A_{\Omega_{2}}\right)\right| \\
& \leq\left|\frac{s_{k}\left(B_{\Omega_{1}}\right)}{\lambda_{k}\left(A_{\Omega_{1}}\right)}-1\right| \lambda_{k}\left(A_{\Omega_{1}}\right)+c_{2} \lambda_{k}\left(A_{\Omega_{1}}\right) \varepsilon+\left|\frac{s_{k}\left(B_{\Omega_{2}}\right)}{\lambda_{k}\left(A_{\Omega_{2}}\right)}-1\right| \lambda_{k}\left(A_{\Omega_{2}}\right) \\
& \leq c_{6}\left(\left|\frac{s_{k}\left(B_{\Omega_{1}}\right)}{s_{k}\left(A_{\Omega_{1}}\right)}-1\right|+\varepsilon+\left|\frac{s_{k}\left(B_{\Omega_{2}}\right)}{s_{k}\left(A_{\Omega_{2}}\right)}-1\right|\right) k^{2 / n},
\end{aligned}
$$

where $c_{6}=\max \left\{c_{2}, c_{3}\right\}$.

The statement follows since by Corollary 7.1

$$
\frac{s_{k}\left(B_{\Omega_{1}}\right)}{s_{k}\left(A_{\Omega_{1}}\right)}=\frac{s_{k}\left(B_{\Omega_{1}}\right) k^{-2 / n}}{s_{k}\left(A_{\Omega_{1}}\right) k^{-2 / n}} \quad \text { and } \quad \frac{s_{k}\left(B_{\Omega_{2}}\right)}{s_{k}\left(A_{\Omega_{2}}\right)}=\frac{s_{k}\left(B_{\Omega_{2}}\right) k^{-2 / n}}{s_{k}\left(A_{\Omega_{2}}\right) k^{-2 / n}}
$$

converge as $k \rightarrow \infty$ to 1 uniformly with respect to $A_{\Omega_{1}}, A_{\Omega_{2}} \in \mathfrak{A}(\mathcal{A})$ and $B_{\Omega_{1}}, B_{\Omega_{2}} \in$ $\mathfrak{B}(\mathcal{A})$.

Concluding remarks. A natural question arises related to the statements of Theorems 6.1 and 7.1. Is the assumption $s>2 l(1-1 / n)$ sharp? We conjecture that it is.

In this paper we are dealing with linear operators in Hilbert spaces. It is of interest to extend the results of the paper to the non-Hilbert case and to some classes of nonlinear operators.

\section{REFERENCES}

[1] Allakhverdiev D.E. On the rate of approximation of completely continuous operators by finitedimensional operators, Uch. Zap. Azerb. Univ. 1957. Vol. 2. P. 27-35.

[2] Burenkov V.I. Sobolev spaces on domains. Stuttgart: B. G. Teubner, 1998. (Teubner-texte zur mathematik; Vol. 137). MR1622690 (99g:46040)

[3] Burenkov V.I., Lamberti P.D. Spectral stability of Dirichlet second order uniformly elliptic operators, J. Diff. Equations. 2008. Vol. 244, No. 7. P. 1712-1740. MR2404437 (2009f:35242)

[4] Burenkov V.I., Otelbaev M. On singular numbers of correct restrictions of non-selfadjoint elliptic differential operators, Eurasian Math. J. 2011. Vol. 2, No. 1. P. 145-148. MR.2910827

[5] Fan K. On a theorem of Weyl concerning eigenvalues of linear transformations. I, Proc. Nat. Acad. Sci. USA. 1949. Vol. 35, No. 11. P. 652-655. MR0034519 (11:600e)

[6] Fan K. On a theorem of Weyl concerning eigenvalues of linear transformations. II, Proc. Nat. Acad. Sci. USA. 1950. Vol. 36, No. 1. P. 31-35. MR0033981 (11:526a)

[7] Fan K. A minimum property of the eigenvalues of completely continuous operators. Eigenvalues of a sum of hermitian matricies, Amer. Math. Monthly. 1953. Vol. 60, No. 1. P. 48-50.

[8] Gohberg, I. C.; Kreın, M. G. Introduction to the theory of linear non-selfadjoint operators. Translated from the Russian by A. Feinstein. Translations of Mathematical Monographs, vol. 18, American Mathematical Society, Providence, RI, 1969, 378 pp. MR.0246142 (39 \#7447)

[9] Kokebaev, B. K., Otelbaev, M., Shynybekov, A. N. On questions of extension and restriction of operators. (Russian) Dokl. Akad. Nauk SSSR 271 (1983), no. 6, 1307-1310. MR0722342(86b:47001)

[10] Lions J.-L., Magenes E. Problèmes aux limites non homogènes et applications. Vol. 2, Paris: Dunod, 1968. (Travaux et recherches mathématiques; No. 18). MR0247244 (40:513) 
[11] Otelbaev, M., Shynybekov, A. N. Well-posed problems of Bitsadze-Samarskiŭ type. (Russian) Dokl. Akad. Nauk SSSR 265 (1982), no. 4, 815-819. MR670839 (84a:47080)

[12] Triebel H. Approximation numbers in function spaces and the distribution of eigenvalues of some fractal elliptic operators, J. Approx. Theory. 2004. Vol.129, No. 1. P.1-27. MR2070178 (2005c:46044)

Faculty of Natural Sciences, People's Friendship University of Russia, Moscow, Russia

E-mail address: burenkov@cf.ac.uk

Faculty of Mechanics and Mathematics, L. N. Gumilyov Eurasian National University, Astana, KazakHSTAN

E-mail address: otelbaevm@mail.ru

Originally published in English 